\title{
Cadena de valor económico del vino de Baja California, México ${ }^{1}$
}

\section{The economic value chain of wine in Baja California, Mexico}

\author{
Salvador González Andrade*
}

Resumen

La cadena de valor se encuentra en el centro de los debates de las políticas económicas en los últimos años debido a la necesidad de incrementarla y cuantificarla. El objetivo de este trabajo es caracterizar la cadena de valor de las empresas vitivinícolas de Baja California sobre la base de los subsistemas organizacionales: gerencia, mercadeo, calidad, productividad y ambiente. A partir de un estudio documental y dos de campo de tipo estadístico descriptivo, se identificaron las capacidades de la empresa y del sector en el cual ésta participa. La cadena muestra que los principales problemas se encuentran en los segmentos relacionados con las endebles infraestructuras productivas, en la falta de proveedores locales de insumos, el intermediarismo en la comercialización y desabasto del producto. Es necesario invertir en capital humano para aumentar la formación y capacitación de los recursos humanos; se identificó la insuficiencia en financiamiento, escasez de agua y elevados impuestos en el sector.

Palabras clave: competitividad, innovación, clúster vitivinícola, agroindustria alimentaria, cadena de valor.

\section{Abstract}

Recent economic policy debates have centered on increasing and quantifying the value chain, and the objective of this research is to characterize the value chain of viticulture in Baja California according to organizational subsystems, including management, marketing, quality, productivity and environment. This study presents a comparative analysis between the capabilities of the vitiviniculture industry and sectors within which it participates. The analysis shows that the primary issues are related to sectors that have weak productive infrastructure, marketing problems, product shortages and few local suppliers, and additional problems include insufficient funding, water shortages and high taxes. Moreover, investments in human capital are required to train human resources.

Keywords: competitiveness, innovation, wine cluster, agro-food industry, horizontal flowchart value chain.

Recibido: 10 de diciembre de 2013.

Aprobado: 19 de noviembre de 2014.

${ }^{1}$ Este trabajo es una versión actualizada de la Cadena de valor de vino en la valles vitivinícolas de Baja California. El estudio es uno de ocho reportes técnicos derivados del "Plan de acción para la innovación y competitividad de los Valles Vitivinícolas de Baja California”, proyecto financiado por el Conacyt a través del Fordecyt. El autor fungió como responsable técnico.

* El Colegio de la Frontera Norte, Departamento de Economía. Dirección: Carretera Escénica Tijuana-Ensenada, km 18.5, San Antonio del Mar, C.P. 22560, Tijuana, Baja California, México. Correo electrónico: salvador@colef.mx 


\section{Introducción}

En el marco actual de la globalización económica, tanto a nivel de naciones como a nivel de regiones, es una constante la búsqueda de aumento de la competitividad. Particularmente en México, el sector vitivinícola presenta amplias perspectivas de crecimiento, pues se estima un aumento en el consumo nacional de vino de $12 \%$ promedio anual para los próximos años. La producción nacional de vino apenas alcanza a cubrir alrededor de $30 \%$ de la demanda en el país y la balanza comercial respecto al vino tiene un déficit de 181.3 millones de dólares; en contraste, Chile tiene un superávit de 1000892 millones de dólares (datos al 2011, Food and Agriculture Organization of the United Nations [FAO], 2013).

No obstante lo anterior, el vino de Baja California, entidad en la que se concentra alrededor de $90 \%$ de la producción nacional, tiene una calidad internacional reconocida (con más de 400 premios y reconocimientos internacionales, de acuerdo con el Consejo Mexicano Vitivinícola, 2010). En dicha entidad la superficie cultivada de la vid ocupa el décimo lugar con 3735.38 hectáreas (un tercio del potencial disponible), alrededor de $90 \%$ de la producción de uva se destina a la elaboración de vino. En particular, los valles vitivinícolas de Baja California se ubican en los municipios de Ensenada, Tecate y Tijuana. Tan sólo en Ensenada se ubican 166 de las 195 unidades de producción de vid de la entidad y ese municipio concentra $90 \%$ de la superficie cultivada de la entidad en las regiones de Valle de Guadalupe, San Vicente, Santo Tomás, Ejido Uruapan, San Antonio de las Minas, entre otras. De hecho, en dichos valles se produce todo el vino de la entidad pero además la superficie cultivada de vid supera a la suma de la extensión de similar propósito de Colima, Nayarit, Aguascalientes y Morelos. Adicionalmente, las condiciones climáticas —bajos niveles de precipitación y la escasez de agua-y las condiciones de cultivo determinan bajos rendimientos, ${ }^{2}$ inferiores a 10

${ }^{2}$ La falta de agua es una de las principales limitantes, de acuerdo con el Sistema Producto Vid de Baja California (spv, s.f., p. 7), el desarrollo sustentable de la vitivinicultura en el Valle de Guadalupe de Ensenada Baja California debe atender la siguiente problemática: poca disponibilidad de agua en el valle, precipitaciones pluviales reducidas, creciente demanda de agua de la población urbana y la sobreexplotación de los acuíferos. 
toneladas de uva por hectárea, pero conceden a la uva mayores niveles de concentración de azúcares y mejores características físicas y químicas, cualidades apreciadas para producir vinos de alta calidad. De ahí la relevancia de aumentar la competitividad del sector analizando la estructura de la cadena de valor de las casas comerciales productoras de vino de la región.

El objetivo del trabajo es describir la cadena de valor del vino, lo cual permite identificar los principales problemas que enfrentan los pequeños y medianos productores. Este es el primer paso a seguir para proponer mecanismos, técnicas y estrategias para reducir los costos de producción, aumentar la diferenciación y para ampliar los mercados de las casas comerciales de vino de los valles vitivinícolas de Baja California.

La metodología empleada consistió en una revisión de la literatura, se aplicaron dos encuestas que permitieron definir la típica cadena o el esquema de flujo horizontal de la cadena de valor del vino, se estimaron los costos de producción de una botella de vino y se analizó la distribución del vino en el estado. Después se construyeron los esquemas gráficos de las cadenas de valor empíricas. Finalmente, con el análisis de la información anterior se identificaron algunos de los principales problemas de los vitivinicultores, destacando las endebles infraestructuras productivas, la falta de proveedores locales, en comercialización existe el intermediarismo y problemas de abasto, la insuficiente formación y capacitación de recursos humanos, el financiamiento insuficiente, la escasez de agua, la alta carga de impuestos, entre otros.

El documento se estructura de la siguiente forma: en el primer apartado se despliega el marco teórico de la cadena de valor y su relación con el desarrollo regional. En el segundo se aborda el contexto en el que se describen las principales empresas de la región de los valles vitivinícolas. Luego, un tercer apartado señala la metodología seguida en el levantamiento de las dos encuestas que recaban información de la producción y distribución del vino. En el cuarto apartado se presentan un diagrama modelo y tres tipos básicos de cadenas de valor, se analizan los costos de producción de una botella de vino, las ventas y distribución de éste; también se discuten los principales problemas enfrentados por los productores de vino y termina con una serie de conclusiones. 


\section{La cadena de valor y su impacto con el desarrollo económico}

\section{Cadena de valor}

Inicialmente Porter (1985) propone que todo el análisis relativo a las fuentes de ventajas competitivas debía de realizarse a nivel de cada una de las actividades que lleva a cabo una empresa a la hora de diseñar, producir, realizar el mercadeo, la entrega o el apoyo de su producto y no a nivel global. Este autor señala que cada empresa tiene una cadena de actividades que aportan un valor a sus clientes, y que es sólo mediante el análisis minucioso y profundo de esta "cadena” que una compañía podrá encontrar fuentes de las ventajas competitivas sostenibles en el largo plazo. Propone que los directivos de las empresas han de reconducir el análisis estratégico detallado hacia la cadena de valor para después poder elegir la mejor estrategia. La cadena de valor "genérica" de todas las empresas se compone de actividades primarias y secundarias; las primeras son las implicadas en la producción del producto, su venta o entrega al comprador y la asistencia posterior a la venta. Las actividades secundarias o de apoyo son las actividades que dan soporte a las actividades primarias y se apoyan entre sí, proporcionando insumos, tecnología, recursos humanos y varias funciones de la empresa. De acuerdo con Porter, son cinco actividades primarias y cuatro actividades secundarias, como se muestra en la figura 1 .

La cadena de valor comprende desde el proveedor hasta el cliente; en ella se realzan las relaciones entre la empresa y sus proveedores. Es una metodología que permite dividir las actividades estratégicas que realiza la empresa para entender el comportamiento de los costos y las fuentes de diferenciación, con el fin de reducir los primeros o de aumentar las fuentes de diferenciación, entendidas éstas como la diferencia que una empresa establece al proporcionar algo único y que es valioso para los compradores más allá de ofrecer un precio bajo. Dicho en otras palabras, la cadena de valor está orientada a satisfacer demandas del mercado, a crear productos diferenciados de mayor valor y un compromiso compartido para cumplir con los requisitos del mercado en cuanto a: calidad, trazabilidad, volúmenes y frecuencia. Posee un flujo de información y conocimiento extensivo; relaciones de coordinación, roles y reglas de juego claramente definidas. En el mismo sentido, en el proceso de creación y consumo de un producto 
Figura 1. Cadena de valor "genérica" de Porter

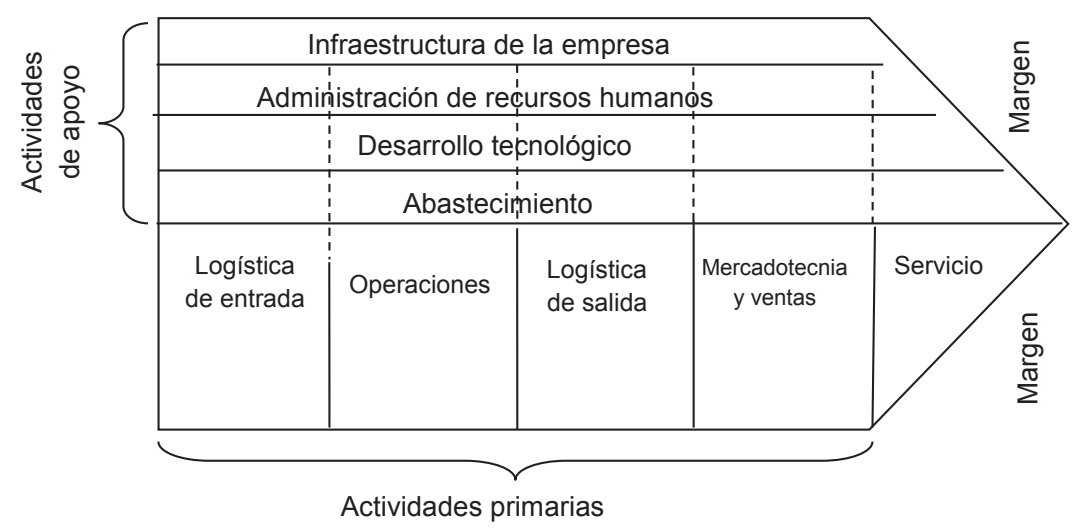

Fuente: Porter, 1985.

se realizan actividades que van añadiendo valor; ese proceso puede visualizarse como un sistema de valor complejo, por ello pueden identificarse al menos cuatro cadenas de valor: de los proveedores, de la empresa, de los canales y de los clientes. ${ }^{3}$

El análisis de la cadena de valor es importante porque las ventajas competitivas se crean a partir de la diferenciación del producto y de la reducción de costos. Para lograr obtener dichas ventajas son necesarios el uso de modernas tecnologías, la innovación y los factores especializados. Estos últimos surgen de habilidades específicas derivadas del sistema educativo, del legado exclusivo del "saber cómo" tecnológico, de la infraestructura especializada, de la investigación, de la capacitación de los recursos humanos, de mercados de capitales desarrollados y de una alta cobertura de servicios públicos de apoyo, entre otros. En el actual contexto caracterizado por los mercados globales y una amplia apertura comercial en México, donde los productores mexicanos compiten con una gran cantidad de productos de procedencia extranjera, algunos de los cuales tienen precios bajos, tal aumento en la oferta nacional redunda en una

${ }^{3}$ Cada actividad de valor emplea insumos, recursos humanos, algún tipo de tecnología para desempeñar su función; adicionalmente cada actividad de valor utiliza y crea información, por ejemplo datos del comprador, parámetros de desempeño de maquinaria y equipo, estadísticas de calidad del producto, entre otras. 
menor demanda de los productos nacionales, por lo que es urgente profundizar la diferenciación de los productos.

\section{Desarrollo económico regional:}

\section{La cadena de valor y la competitividad}

El desarrollo económico no solamente se sustenta en la dotación y en el uso eficiente de los recursos y en el potencial que esos representan. Además, toma en cuenta la organización de la producción, la difusión de conocimientos, entre otros atributos que sirven para definir el nivel alcanzado (Vázquez, 2005, p. 38). En particular, el desarrollo endógeno incorpora un enfoque territorial en el que las actividades productivas se realizan con los recursos disponibles. También se busca satisfacer las necesidades y demandas locales por medio de la participación de la comunidad local. En ese sentido, se le considera una estrategia más integral, no es simplemente el mejoramiento de los sistemas productivos locales en el ámbito laboral nacional o internacional, sino también pretende lograr un aumento en el bienestar económico, social y cultural de toda la población (Vázquez, 2002, p. 22). ${ }^{4}$

La competitividad tiene como finalidad la sostenibilidad de las actividades productivas. Dicho concepto es importante en el desarrollo y mejoras de las fortalezas o capacidades de las cadenas productivas en una determinada región. ${ }^{5}$ La competencia no solamente se da entre empresas o fábricas individuales, sino entre sistemas enteros de producción, cadenas productivas o cadenas de valor. Esto hace que se formen alianzas entre agentes de producción independientes (proveedores, productores) para formar un sistema de relaciones empresariales teniendo confianza y

${ }^{4}$ Los sistemas productivos locales son un modelo basado en la división del trabajo entre empresas y con la creación de un sistema de intercambios locales trae como resultado un incremento de la productividad y crecimiento de la economía (Vázquez, 2005).

${ }^{5}$ El concepto de competitividad inicialmente fue usado únicamente para comparar países usando indicadores macroeconómicos, sin embargo, durante 1991, Porter sugirió que se aplicara también para comparar empresas o productos semejantes que compiten en los mercados, ya sea nacionales o internacionales (Girán, Vargas y Osta, 2008). 
conocimiento mutuo entre ellos y que se anticipen a los cambios del entorno en el cual se desenvuelven (Vivanco, Martínez y Taddei, 2010, p. 170).

Qi, Zhao y Sheu (2011) analizan la relación entre la estrategia competitiva, la estrategia de cadena de suministro y los resultados empresariales o la evolución en los negocios al examinar los efectos moderadores de la incertidumbre del entorno. Ello implica coalinear la estrategia de la cadena de suministro y la estrategia competitiva — por diferenciación de productos o por líderes en precios-con el entorno para mejorar el rendimiento.

De igual manera, Moguillansky, Salas y Cares (2006) aportan elementos para mejorar las políticas e instrumentos que aseguran la competitividad, para ello realizaron una evaluación de la capacidad para innovar de dos cadenas alimentarias en Chile, una de ellas es la cadena del vino. Analizaron la competitividad de la empresa a partir del comportamiento de la misma estudiando el contexto en que ésta se desenvuelve, principalmente las características de la cadena de valor, el entorno económico y el mercado, así como el desempeño en la innovación de los principales competidores del mercado mundial. Después construyeron un modelo del sistema de innovación, el cual se concentró en siete focos de análisis interrelacionados: la estrategia empresarial, las prácticas innovadoras al interior de la empresa, la articulación a redes de conocimiento, la alerta tecnológica, disponibilidad de recursos, condiciones de entorno y resultados. Con base en el modelo desarrollaron un instrumental metodológico de recolección de información cualitativa y cuantitativa, en el que incluyeron variables útiles para caracterizar el estado del sistema de innovación en las empresas.

Adicionalmente, en el análisis de un sistema productivo es conveniente incluir la teoría de costos de transacción, pues se apega con realismo y precisión al enfoque de cadenas de valor. ${ }^{6}$ Analiza las alternativas existentes para organizar interrelaciones entre empresas que mejor se adapten a las características de cada intercambio para minimizar riesgos y costos. Todo el sistema involucrado necesita disminuir esos costos teniendo en cuenta

${ }^{6}$ El mercado origina costos que en algunos casos pueden ser evitados cuando las empresas buscan mecanismos para asignar recursos de modo más eficiente. Por ejemplo, el análisis de los costos de producción y de transacción se divide en costos de información y de negociación. Los primeros son útiles en la determinación de precios relevantes, mientras que los costos de negociación incluyen la elaboración y cierre de contratos en cada relación de intercambio. 
el ambiente donde se desarrolla (leyes, reglas, costumbres, sociedad) (Iglesias, 2002).

En la misma línea de discusión, Fischer, Hammond, Obermeyer y Raman (1994) estudiaron el suministro para satisfacer la demanda en un mundo incierto. Indican que la competencia mundial hace cada vez más rápido el desarrollo de productos y de sistemas flexibles de fabricación. Los fabricantes y minoristas pueden reducir considerablemente el coste de los errores de predicción incluyendo una "respuesta precisa", un nuevo enfoque para la predicción de toda la planificación y el proceso de producción. Las empresas pueden mejorar sus previsiones y, simultáneamente, rediseñar sus procesos de planificación para reducir al mínimo el impacto de los pronósticos inexactos. Hacer la cadena de suministro más rápida y flexible, de modo que los administradores puedan posponer decisiones sobre sus elementos más impredecibles hasta que tienen algunas señales del mercado, como los resultados de ventas de principios de temporada, son útiles para ayudar a adecuar correctamente la oferta a la demanda.

Por su parte, Lee (2004) plantea que la cadena de suministro debe responder a conceptos enmarcados en la "triple A": agilidad, adaptabilidad y alineación. La agilidad tiene como objetivo responder a los cambios a corto plazo en la demanda o la oferta rápidamente, manejar interrupciones externas sin problemas; la adaptabilidad busca ajustar el diseño de la cadena de suministro para cumplir con los cambios estructurales de los mercados, modificar la red de estrategias de abastecimiento de productos y tecnologías; mientras que la alineación pretende crear incentivos para un mejor desempeño.

Además, Lee (2010) afirma que para mejorar la sustentabilidad ambiental no es suficiente que las empresas sólo ajusten la cadena de suministro, sino que deben repensar de punta a punta sus procesos. Las empresas en toda la cadena de suministro deberían adoptar un enfoque holístico de la sostenibilidad y perseguir cambios estructurales más amplios. Éstos pueden incluir innovaciones radicales en los procesos de producción, el desarrollo de relaciones fundamentalmente diferentes con los socios de los negocios que pueden evolucionar hacia nuevos modelos de servicio, e incluso de colaboración con varias empresas para crear nuevas estructuras de la industria; reinventar los procesos de fabricación e incluso 
la vinculación con los competidores para afrontar los retos de escala. El resultado puede ser una cadena de suministro más verde que requiere menos capital, a costos de operación mucho más bajos, los cuales le darían una ventaja competitiva.

Finalmente, Sánchez (2002) analiza el modelo de organización de los sistemas productivos vitivinícolas y los efectos que dicha estructura tiene sobre la productividad y la competencia de las empresas en el Valle de Guadalupe en Ensenada.

\section{La vitivinicultura en Baja California}

\section{Las empresas vitivinícolas ${ }^{7}$}

Si bien el cultivo de la vid en Baja California se remonta a la época de la conquista cuando fue introducida por los jesuitas, actualmente Bodegas de Santo Tomás es la única empresa que sigue vigente y se remonta a esa época. Una de las vinícolas de más tradición es L. A. Cetto, fundada en 1930, mientras que la mayoría de las organizaciones que están en el mercado se remontan a las décadas de 1970 y de 1980, tales como Monte Xanic, Chateau Camou, Casa de Piedra, Mogor Badan, Cavas Valmar, Adobe Guadalupe. ${ }^{8}$

Actualmente, por su aportación en la economía estatal, el empleo y cobertura, el cultivo de la vid ocupa el octavo lugar en importancia. En el año 2008 ocupó una superficie sembrada de 3610 hectáreas y la cosechada fue de 2918 ha. En ese año se obtuvo una productividad media de 5.49 toneladas por hectárea y un valor de la producción de 133610000 pesos y generó 1638 empleos (Sepúlveda, 2009, p. 17). Ya en el año 2010 la vid ocupó una superficie sembrada de 3735.38 ha.

${ }^{7}$ En la vitivinicultura se identifican tres tipos diferentes de empresas: las vitícolas se dedican a producir uva, las vinícolas producen vino y las vitivinícolas producen tanto uva como vino.

${ }^{8}$ En la entidad recientemente se han incorporado nuevos productores de la vid, pues 22.33\% (683.32 ha) de las plantaciones de la uva en producción son plantas nuevas que tienen de uno a cinco años (Secretaría de Fomento Agropecuario [Sefoa], 2011, p. 33). 
En Baja California la superficie cubierta por vid se ubica en cuatro de los cinco municipios: Ensenada contribuye con $89.94 \%$ de la superficie plantada (3 359.75 ha), seguida de Mexicali con $7.29 \%$ (272.23 ha), ${ }^{9}$ Tijuana con $1.79 \%$ (66.90 ha) y Tecate con $0.98 \%$ (36.50 ha) (Sefoa, 2011).

De la superficie cultivada en el estado, el producto de 2904.40 hectáreas $(77.75 \%)$ se destina a la elaboración de vino. No obstante, se estima que alrededor de 8000 hectáreas tienen potencial para este cultivo. El inventario es de 46 diferentes variedades plantadas y en producción, las cuales tienen diferentes orígenes, principalmente español, francés e italiano.

En Ensenada la producción de vid se ubica en seis valles vitivinícolas: destaca el Valle de Guadalupe, pues concentra la mayor superficie cultivada de vid para elaboración de vino (46.61\%), en segundo lugar está el Valle de San Vicente (34.19\%), le siguen Santo Tomás (6.9\%), el Ejido Nuevo Uruapan (3.44\%), San Antonio de las Minas (3.34\%), Ojos Negros (1.52\%) y otras localidades $(4.00 \%)$ (Sefoa, 2011, p. 26).

La producción primaria la realizan un gran número de productores, de los cuales 14 tienen un área de cultivo de 50 o más hectáreas, participan con 1250.85 ha de cultivo (46\%); 22 productores pequeños y medianos tienen entre 20 y 49 ha y significan 696.04 ha (28\%); y 132 productores tienen entre 1 y 19 ha y contribuyen con 741.62 ha (26\%) (sPv, 2008).

En el cuadro 1 puede observarse que los costos de producción de uva en la etapa de mantenimiento y en la modalidad de riego ${ }^{10}$ para el año 2009 ascienden a 48405 pesos por hectárea con un rendimiento estimado en 10 toneladas. ${ }^{11}$ Los mayores gastos se deben al control de plagas y a los

${ }^{9}$ En Mexicali las áreas vitícolas pertenecen a la cuenca del Río Colorado y se ubican en su totalidad en el Valle de Mexicali, ahí la viticultura está orientada $100 \%$ a la producción de uva de mesa y pasa.

${ }^{10}$ En la entidad sólo $5.38 \%$ de la superficie de vid es de temporal (Distrito de Desarrollo Rural [DDR], 2011).

${ }^{11}$ Las condiciones climáticas y las condiciones de cultivo de la uva contribuyen para conseguir una producción de calidad. Es importante controlar el rendimiento y el exceso de vigor evitando todos aquellos factores que lo favorecen: riego, abonado nitrogenado excesivo, marcos anchos de plantación, porta-injertos vigorosos, etc. Asimismo, es importante la elección adecuada de la fecha de vendimia y una esmerada elaboración para conseguir vinos de calidad. Los valles vitivinícolas de Ensenada se caracterizan por los bajos niveles de precipitación y por la escasez de agua en la región, ello contribuye a obtener rendimientos inferiores a 10 toneladas de uva por hectárea. 


\section{Cuadro 1. Costo medio de producción por hectárea de uva en la etapa de mantenimiento y en la modalidad de riego, Baja California, ciclo 2009}

\begin{tabular}{|lrr|}
\hline \multicolumn{1}{|c}{ Concepto } & Costo $(\$)$ & Por ciento \\
\hline Labores de fertilización & 6040 & 12.48 \\
Sistema de riegos/Aplicación de riegos & 10664 & 22.03 \\
Labores culturales & 3480 & 7.19 \\
Control de plagas & 18711 & 38.66 \\
Cosecha & 5390 & 11.14 \\
Poda en producción & 4120 & 8.51 \\
\multicolumn{1}{|c|}{ Total } & 48405 & 100.00 \\
\hline
\end{tabular}

Notas: 1. Se estima un rendimiento esperado de 10 toneladas por hectárea.

2. Las variedades Sirah, Merlot, Cabernet Suavignon y Tempranillo tienen menores requerimientos de fertilizantes.

Fuente: Elaboración del autor con base en DDR (2011).

sistemas y aplicación de riegos; en menor cuantía se encuentran los gastos de las labores de fertilización, cosecha, poda y las labores culturales (DDR, 2011).

\section{Producción vinícola}

Son alrededor de 57 las casas productoras de vino, la mayoría organizadas en el Sistema Producto Vid en Baja California (SPV) con 47 socios y 10 empresas no están agremiadas. ${ }^{12}$ Destacan las casas vinícolas: Ácrata,

De ahí que la pulpa de las uvas se caracteriza por sus elevadas concentraciones de azúcares o por su bajo contenido de agua; en contraparte, en Chile el rendimiento de diversas variedades de uva empleadas para producir vino llega a superar las 20 toneladas por hectárea.

${ }^{12}$ En el SPV participan productores de vid, casas vinícolas, proveedores de insumos y equipos, comercializadoras de uva o vino, organizaciones afines (Canirac, Canacintra, Proturismo), instituciones financieras de desarrollo (FIRA, FIRCO, Finrural y comercial Banorte y Bancomer), centros educativos y de investigación, instituciones 
Adobe Guadalupe, Alborada Guadalupe, Barón Balché, Bibayoff, Bodegas de San Antonio, Bodegas de Santo Tomás, Casa de Piedra, Casa Domecq, Cavas Valmar, Château Camou, Jalá, L. A. Cetto, Mogor Badan, Monte Xanic, Roganto, Viña de Liceaga, Vinisterra, entre otras.

La producción de vino es de nueve millones de litros, es decir, un millón y cinco mil cajas de nueve botellas cada una. De acuerdo con los últimos datos del SPV (2008), la producción por tamaño de empresa es como sigue: hay tres casas comerciales grandes que concentran $82 \%$ de la producción del vino de la región (821 000 cajas); otras tres son empresas medianas que tienen una producción de 120000 cajas (12\%). Dichas empresas coexisten con otras 25 casas comerciales pequeñas que emplean técnicas artesanales en la producción y tienen un volumen anual de producción de 59000 cajas de vino (5\%). En un cuarto grupo se ubican 26 microempresas que producen 5000 cajas (1\%). En la elaboración de la bebida estas empresas combinan técnicas artesanales y modernas, maquilando algunas partes de sus procesos.

$\mathrm{Al}$ igual que las variedades, las "escuelas" enológicas que participan son de diversos orígenes: español, italiano, francés, chileno, argentino y suizo. En Ensenada 83.18\% de la superficie (2 794.75 ha) se destina a la producción de uva para vino, el resto es de uva de mesa. Siete variedades de vino ocupan más de la mitad de la superficie, destacan Cabernet Sauvignon (20.23\%), Chenin Blanc (6.77\%), Merlot (6.69\%), Tempranillo (5.43\%), Nebbiolo (5.26\%), Grenach (4.14\%) y Chardonnay (4.04\%).

Con relación a la competitividad, históricamente el precio aparente de exportación del vino mexicano ha seguido una tendencia constante al alza, al pasar de alrededor de medio dólar por litro en las décadas de 1960 y 1970, hasta alcanzar los 4.43 dólares por litro en la actualidad. Dicho aumento en el precio medio de exportación refleja una orientación hacia un mercado de vinos de mayor calidad.

El diferencial en los precios internacionales del vino de exportación ubica a España y Chile como los principales competidores de México: ostentan un precio sumamente bajo de 1.33 y 2.55 dólares por litro de vino, respectivamente. En menor cuantía, los vinos mexicanos también compiten por precio con Italia, Alemania y Portugal. El cuadro 2 muestra los precios

de gobierno federal y estatal (Sagarpa, Sefoa, Sectur, Sedeco) y el ayuntamiento de Ensenada (sPV, 2008). 
medios de los vinos para exportación de México y de sus principales competidores en diversos periodos.

A su vez, Argentina habitualmente ha seguido un comportamiento voluble en materia de precios, pero manteniendo siempre precios relativos menores respecto a los de México, Estados Unidos y hasta 1999, respecto a los de Chile. En el continente europeo tanto España como Italia han mantenido una política de precios relativos bajos respecto a México. Actualmente los vinos mexicanos son cerca de tres veces más caros que los de España, y son cerca de dos veces el precio de los vinos italianos, mientras que los precios de los vinos alemanes son alrededor de un tercio más

\section{Cuadro 2. Precios aparentes de los vinos para exportación en países seleccionados, 1961, 1970, 1980, 1986, 1990, 2010 y 2011 (valuados a precios fob, dólares por litro)}

\begin{tabular}{|l|c|c|c|c|c|c|c|c|c|c|}
\hline País / Año & 1961 & 1970 & 1980 & 1986 & 1990 & 2000 & 2005 & 2010 & 2011 & $\begin{array}{c}\text { Relación } \\
\text { 2010/2000 }\end{array}$ \\
\hline México & 0.48 & 0.59 & 0.75 & 0.73 & 1.70 & 2.80 & 2.27 & 4.53 & 4.43 & 1.62 \\
\hline Chile & 0.17 & 0.38 & 1.30 & 1.09 & 1.20 & 1.43 & 2.13 & 2.11 & 2.55 & 1.47 \\
\hline Argentina & 0.50 & 0.22 & 0.89 & 0.32 & 0.38 & 1.61 & 1.37 & 2.62 & 2.66 & 1.62 \\
\hline $\begin{array}{l}\text { Estados } \\
\text { Unidos }\end{array}$ & 0.63 & 0.87 & 1.02 & 1.31 & 1.35 & 1.92 & 1.79 & 2.75 & 3.19 & 1.44 \\
\hline Europa & 0.27 & 0.38 & 1.09 & 1.38 & 2.17 & 2.22 & 2.89 & 2.99 & 2.08 & 1.35 \\
\hline Alemania & 0.83 & 0.87 & 1.86 & 1.60 & 1.78 & 1.46 & 2.36 & 2.96 & 3.28 & 2.03 \\
\hline España & 0.19 & 0.20 & 0.70 & 0.78 & 1.31 & 1.45 & 1.39 & 1.38 & 1.33 & 0.96 \\
\hline Francia & 0.36 & 0.70 & 1.96 & 2.07 & 3.46 & 3.40 & 5.13 & 5.95 & 6.49 & 1.75 \\
\hline Italia & 0.19 & 0.22 & 0.56 & 0.73 & 1.19 & 1.52 & 2.40 & 2.36 & n. d. & 1.55 \\
\hline Portugal & 0.16 & 0.32 & 1.50 & 1.67 & 2.70 & 2.50 & 2.60 & 3.19 & n. d. & 1.28 \\
\hline
\end{tabular}

Notas: 1) Precios aparentes definidos como el cociente entre el valor de las exportaciones y el volumen de las exportaciones; 2) Chile, en 1980, tuvo un valor atípico alto; años antes y después los precios aparentes estuvieron por debajo de 0.91 dólares por litro; 3) México, las devaluaciones tuvieron marcados efectos de abaratamiento de las exportaciones en 1986 y en 2002; 4) precios FOB: Es el precio libre a bordo (FOB) de las mercancías puestas en un puerto de carga. Significa el precio acordado al cual el vendedor entrega la mercancía a bordo del buque designado por el comprador en el puerto de embarque; 5) n. d. = no disponible.

Fuente: Elaboración del autor con base en la FAO (2013). 
económicos que los mexicanos. En el lado contrario, únicamente Francia ha seguido una tendencia sistemática y consistente a lo largo de la segunda mitad del siglo pasado y hasta la fecha procura vinos de calidad a precios relativamente altos. Si bien la brecha entre el precio medio de los vinos franceses y mexicanos ha tendido a reducirse, aún en la actualidad los vinos provenientes de Francia son alrededor de $30 \%$ más caros que los mexicanos.

A partir de 1990 los productores nacionales de vino se orientaron al mercado de vino de alta calidad, denominado vino Premium. Esta política de calidad la han mantenido las nuevas empresas y ha conseguido el reconocimiento del vino de Baja California en el mercado como vino de alta calidad; a la fecha la entidad cuenta con más de 300 premios y reconocimientos internacionales. ${ }^{13} \mathrm{El}$ mercado nacional está reconociendo la calidad del vino nacional y se está logrando paulatinamente ganar terreno a los vinos importados, especialmente de Europa.

No obstante lo anterior, la participación actual del vino mexicano en el mercado nacional es de sólo 30\%, el resto del mercado lo cubre la producción importada, caracterizada por vinos de bajo precio, menor a 100 pesos, en la que destacan los vinos chilenos. La viticultura en Baja California tiene valiosas oportunidades: primera, de acuerdo con las estimaciones del Consejo Mexicano Vitivinícola (2012), el mercado del vino de mesa ha venido creciendo a ritmos de $12 \%$ promedio anual durante la última década (una expectativa conservadora es que al menos dicha tendencia se mantendrá por varios años más); segunda, además es factible participar en una mayor proporción del mercado nacional.

\section{Metodología}

Se consideran algunos elementos de la propuesta metodológica de Lundy, Gottret, Cifuentes, Ostertag y Best (2004), quienes formulan un proceso a seguir para construir diversas estrategias para aumentar la competitividad de las cadenas productivas con productores de pequeña escala. Dichos autores también proponen desarrollar grupos de trabajo con los

${ }^{13}$ Solamente en el año 2010 Monte Xanic obtuvo nueve reconocimientos, L. A. Cetto ocho, Chateau Camou dos, Santo Tomás uno y Viña de Liceaga uno. 
productores para identificar fortalezas y debilidades de la cadena de valor y para generar estrategias para elevar la competitividad.

En el presente estudio, además de las entrevistas sostenidas con informantes clave, se realizó trabajo de campo para levantar dos encuestas: la primera en el 2011 recoge información de 25 pequeños y medianos vitivinicultores; la segunda en el 2012 se aplicó a 24 distribuidores de vino de la región ubicados en la entidad. Dicha información sirvió para construir la estructura genérica (diagrama horizontal) y empírica o individual de las cadenas de valor en las empresas vinícolas; estimar los costos de producción de una botella de vino artesanal para analizar la distribución del vino e identificar la problemática existente del sector vitivinícola.

\section{Identificación de la cadena de valor de los vitivinicultores de Baja California}

Con base en el padrón de productores registrados en el SPV, se seleccionó una muestra aleatoria de 25 vitivinicultores pequeños y medianos ubicados en el municipio de Ensenada (aproximadamente 40\% de los 57 vinicultores del padrón 2008). A los productores seleccionados, en el año 2011 se les aplicó un cuestionario para identificar la cadena de valor de la empresa. Las preguntas centrales de investigación fueron: ¿Es posible reducir los costos de producción del vino sin afectar la calidad? ¿Cuáles son los segmentos de la cadena de valor donde se enfrentan los mayores problemas para los que sería necesario introducir acciones correctivas? Ello permitió identificar los principales logros, así como la problemática enfrentada en el desarrollo de su actividad. También se determinaron los segmentos competitivos y no competitivos de la cadena de valor de las empresas vitivinícolas.

\section{Encuesta a distribuidoras de vinos de la región}

La segunda encuesta tuvo como objetivo recabar información para analizar el proceso de distribución del vino de la región; se aplicó en junio de 
2012 a 24 distribuidoras localizadas en los municipios de Ensenada (5), Mexicali (5), Tijuana (11) y Playas de Rosarito (3). Dicha encuesta sirvió para estimar la participación del vino mexicano en el mercado doméstico: los rangos de precios de los vinos que más se venden, las principales variedades de uva, también fue útil para identificar los principales problemas en los canales de distribución, así como para trazar algunas soluciones y recomendaciones de los propios distribuidores de vino. ${ }^{14}$

\section{Construcción de la cadena de valor de los vitivinicultores de Baja California}

El proceso de elaboración del vino se ilustra por medio de un diagrama tipo de flujo horizontal de la cadena de valor (figuras 2 a y 2b). En dicho esquema se identifican las etapas principales y secundarias de la cadena de valor del vino, así como al responsable de la etapa. Por ejemplo, al vendimiador, el laboratorista, el operario del Centro de Distribución (CEDIS) y al consumidor. Si bien el diagrama es extenso y detallado, con el objetivo de facilitar su lectura y comprensión se emplean seis íconos, cada uno de los cuales tiene un motivo y un significado especial como se indica en la figura 2a. Por ejemplo, el tomar una decisión sobre qué tipo de vino se produce, si es blanco, rosado o tinto implica seguir un proceso específico, ello hace necesario el tomar una decisión sobre la adición del dióxido de azufre o sulfitado. El tipo de vino a elaborar determina las etapas del proceso a seguir. Por ejemplo, en el tinto de reserva es necesario el encubado en barricas para su oxigenación, no así para algunos vinos blancos o los rosados. De ahí la necesidad de establecer los conectores de proceso (A y B). El ícono de tiempo de espera se emplea únicamente para ilustrar la etapa de envejecimiento y crianza.

${ }^{14}$ Un producto derivado de esta encuesta es un censo con la ubicación de 45 restaurantes que ofrecen a sus comensales vinos de los valles: 19 están en Tijuana, 11 en Mexicali, 11 en Ensenada y cuatro en Playas de Rosarito. El proveer información a residentes y turistas es útil en la promoción del consumo del vino de Baja California (González, 2012, p. 44). 
Costos de producción "artesanal" de una botella de vino

En los costos de producción del vino influyen directamente el tipo de tecnologías empleadas, el volumen de producción, el tipo de vino a elaborar, entre otros factores. Basados en Lagos, Espinoza y Rojas (2010), se estiman los costos de producción de una botella de vino de una " $x$ " variedad en dólares americanos empleando en el proceso una tecnología de tipo artesanal (no industrial de grandes volúmenes). Los precios de los insumos, actualizados al año 2012, se obtuvieron mediante información directa de las compañías distribuidoras, tales como la Distribuidora de artículos para vinícolas y oliveras (Duarte, 2012).

El costo promedio de una botella de vino se estimó de la manera siguiente. Se considera una producción de 500 litros de vino económico o Premium que influye directamente en los costos variables. La estructura de costos tiene tres componentes: 1) los activos, que incluyen gastos preoperativos generales y de inversión en activos fijos en los que se supone una tasa de depreciación promedio anual de $20 \%$; 2) los costos variables,

Figura 2a. Diagrama de flujo horizontal: Íconos y siglas empleados y su significado

\begin{tabular}{|c|c|}
\hline Ícono & Concepto \\
\hline & Responsable de la etapa \\
\hline & Inicio/Fin \\
\hline & Proceso \\
\hline & Decisión \\
\hline & Tiempo de espera \\
\hline A & Conector de proceso \\
\hline CEDIS & \begin{tabular}{c} 
Centro de distribución \\
Modelo clásico de venta: \\
\hline AIDA
\end{tabular} \\
\hline Incoterm & $\begin{array}{c}\text { Acrónimo del inglés } \\
\text { International comercial terms }\end{array}$ \\
\hline
\end{tabular}




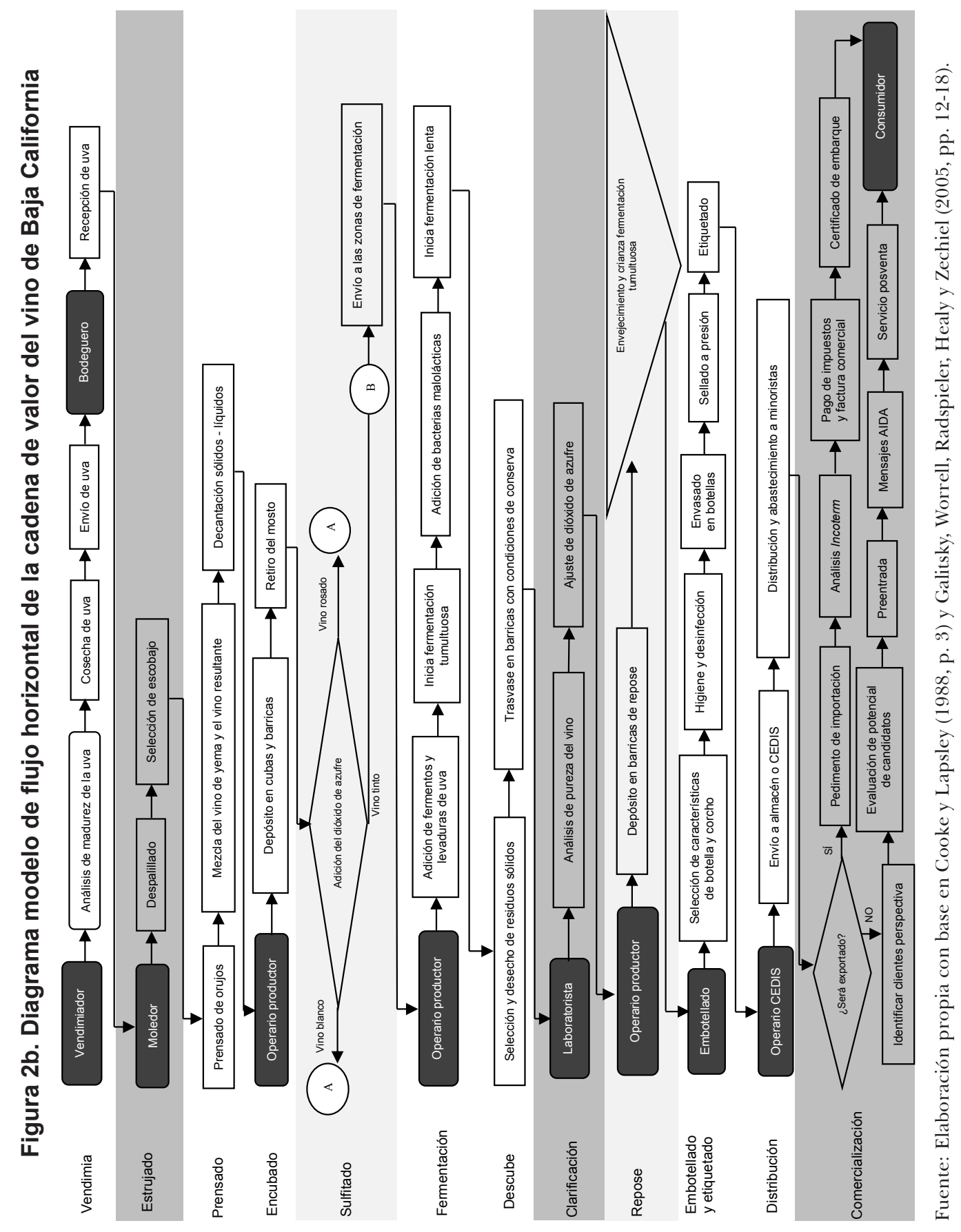


que incluyen materia prima, insumos y mano de obra, y 3) los administrativos, que consideran alquiler, servicios de luz y agua, seguros y los registros.

La crianza o envejecimiento de los vinos es un proceso largo y delicado cuyo objetivo es conferir unos caracteres distintos a un vino. ${ }^{15}$ Para elaborar un vino de calidad Premium, en los costos relacionados con la crianza se considera el uso de barricas por un periodo de 12 meses, con el supuesto de que las barricas se deprecian al 100 por ciento.

Los productores de Baja California emplean barrica bordelesa con una capacidad de 225 litros, por precios y preferencias usan en promedio dos terceras partes las de roble americano y una tercera parte las de roble francés, esa proporción de uso la denominamos barrica "tipo".

\section{Resultados}

\section{Cadena de valor de los vitivinicultores de Baja California}

El diagrama modelo de flujo horizontal de la cadena de valor del vino de Baja California muestra las 12 etapas que se consideran más relevantes en el proceso completo (figura 2b). Dichas etapas se han colocado a manera de columna en el margen izquierdo del diagrama y en su costado derecho, a la misma altura, se colocaron los pasos más sobresalientes durante la etapa correspondiente, así como los elementos o individuos responsables de llevar a cabo dichos procesos. La etapa inicial es la vendimia: el vendimiador inicia el proceso con el análisis de madurez de la uva, le sigue la cosecha y después el envío o transporte al bodeguero, quien recibe la uva. El estrujado es la segunda etapa, el moledor es el responsable del despalillado, de la selección del escobajo. La tercera etapa es del prensado de orujos,

${ }^{15} \mathrm{El}$ proceso de envejecimiento se realiza en dos fases: oxidativa y reductora (sin oxígeno). La primera tiene lugar en la barrica de madera, donde reducidas cantidades de oxígeno penetran en el interior del recipiente modificando de forma natural la estructura química de muchos de los componentes del vino. La segunda fase se realiza en el interior de la botella. En ella no penetra prácticamente oxígeno, a excepción de pequeñísimas cantidades de gases que se filtran a través de las células del corcho, por lo que los elementos del vino reaccionan entre sí en su ausencia. 
la mezcla del vino de yema y de la decantación que consiste en separar los sólidos y líquidos. El encubado es la cuarta etapa, el responsable es el operario productor, quien realiza el depósito en cubas y barricas y el retiro del mosto. La quinta etapa es el sulfitado, le siguen la fermentación, ${ }^{16}$ el descube y otras más que añaden valor hasta llegar al embotellado y etiquetado; después le siguen la distribución y finalmente la comercialización, en donde el responsable en ambas es el operario del ceDis.

En la comercialización se toman decisiones importantes, como la de destinar el producto al mercado nacional o al extranjero. Si el producto se exporta el proceso continuará atendiendo las fases de pedimento de importación, el análisis de los términos del comercio internacional (Incoterm), el pago de impuestos, la elaboración de factura comercial y la obtención de un certificado de embarque antes de arribar a las manos del consumidor final. De otra manera, si el producto es orientado al mercado nacional el proceso continuará cubriendo las fases de identificación de clientes, la evaluación de candidatos, el trabajo de preentrada, los mensajes de atención, interés, deseo y acción (AIDA) así como los servicios de posventa que se brindan al consumidor final.

${ }^{16}$ La fermentación es una reacción química compleja y al mismo tiempo un proceso totalmente natural. Las uvas fermentan cuando la piel de los granos se rompe: los azúcares contenidos en el interior de los frutos maduros entran entonces en contacto con las levaduras presentes en el hollejo que recubre cada grano y comienza la fermentación. La fermentación es un proceso fuertemente exotérmico, liberándose $23,5 \mathrm{kcal}$ por cada mol de glucosa consumida. Además de la muerte de las levaduras, una temperatura elevada produce la pérdida de compuestos volátiles aromáticos y un incremento en la extracción de taninos y sustancias amargas provenientes de las partes sólidas presentes. El control de la temperatura puede lograrse mediante un sistema adecuado de refrigeración, para que las mismas puedan acotarse a los rangos deseados por el vinicultor. El proceso de fermentación suele dividirse en tres etapas: 1. Prefermentación, es el conjunto de operaciones anteriores a la fermentación; 2. Fermentación, y 3. Postfermentación, es el conjunto final de operaciones, aquí ocurre la fermentación lenta (Ponce, 2009). Mendoza (2011, p. 11), al referirse al periodo de posfermentación, incluye la maceración larga. Plantea que el encubado prolongado afecta a la evolución de los taninos y crea más cuerpo, complejidad, profundidad en el paladar y aumenta la estabilidad del color. Es posible que también aumente la tasa de norisoprenoides, precursores aromáticos ubicados en células epidérmicas del hollejo. 


\section{Cadenas de valor del vino empíricas}

En las vitivinícolas pequeñas y medianas encuestadas se emplea fundamentalmente el trabajo familiar. En tres cuartas partes de ellas solamente tienen un empleado, y en cerca de $19 \%$ de ellas tienen de cuatro a seis empleados. Adicionalmente, algunas de las empresas cuentan con asesores especialistas como ingenieros y/o enólogos en menor cuantía. En pocas de ellas, y sólo a una parte de los trabajadores, se les brindan cursos de capacitación y promoción de la cultura del vino.

Los productores de vino de Baja California se agrupan en tres tipos: viticultores, vinicultores y vitivinicultores. La elaboración de la cadena de valor segmentada por tamaño de productores permite identificar el conjunto de factores internos a la empresa que determina la posición en el mercado (abastecimiento, desarrollo tecnológico, recursos humanos, infraestructura de la empresa, operaciones, logística, marketing y ventas, etc.). Su correcta interpretación permite identificar qué actividades de la empresa crean valor y cuáles sólo ocasionan pérdidas a la compañía. A partir de los resultados de la encuesta a vitivinicultores se construyen tres esquemas gráficos (ver figuras 3 a la 5), que muestran de manera agregada la cadena de valor del vino. La figura 3 despliega los cuatro eslabones principales de la cadena; de hecho es factible el análisis de los eslabones construyendo una cadena de valor para cada uno de ellos. La figura 4

\section{Figura 3. Cadena de valor vitivinícola: Esquema general con cuatro cadenas}

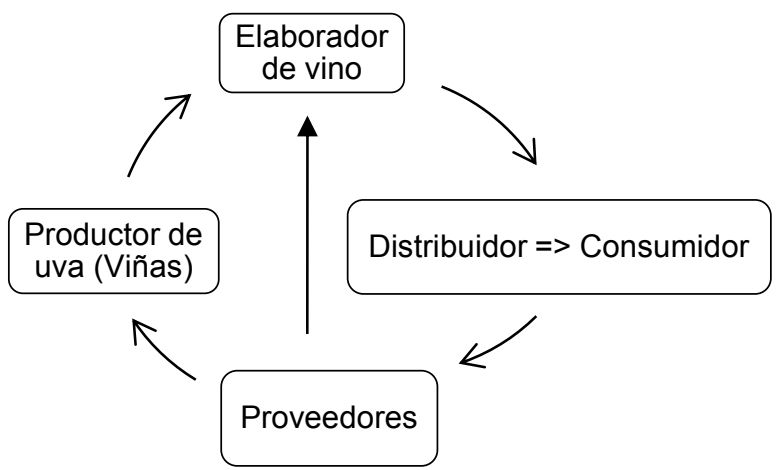


exhibe algunas de las relaciones entre productor, consumidor e intermediarios.

En particular, la figura 5 presenta el proceso básico de transporte que sigue el vino desde la cava o fabricante hasta el consumidor final. A partir del diagrama básico se pueden encontrar diversas variantes y combinaciones en función del volumen de producción y de los canales de comercialización seguidos en las empresas. En cavas pequeñas y medianas el proceso de transporte puede incluir múltiples combinaciones de etapas o de intermediarios que sigue el producto desde la cava hasta el consumidor final. En el caso de las cavas medianas y grandes el producto puede seguir

Figura 4. Cadena de valor del vino: Productor-consumidor e intermediarios



Figura 5. Cadena de valor del vino: Productor-consumidor final, transporte físico

Agrilcultor,

Cava,

Bodega

vinícola

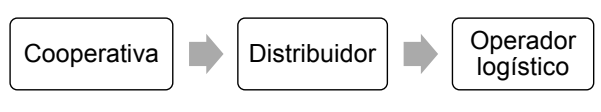

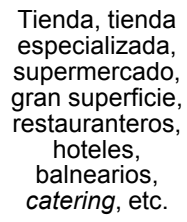
especializada, supermercado, gran superficie, restauranteros, hoteles, balnearios, catering, etc.

Consumidor final 
rutas que incluyen un operador logístico, o bien, el proceso puede ser más simple y unir directamente al productor con el consumidor final. Algunos productores se apoyan en la promoción y ventas virtuales mediante el uso de la web e internet. Al vender en formas diferentes al final de la cadena se tiene el control; al aumentar los canales comerciales se aumentan las posibilidades de venta. El canal directo cava-consumidor final sería el más deseable y recomendable, pues permite elevar el ingreso de los productores de vino. Si bien el enlace directo es el más eficiente, no se deben dejar de lado los demás caminos.

\section{Costos de producción artesanal de una botella de vino}

El proceso de producción artesanal de vino implica al menos tres grupos de costos: activos totales (incluye gastos preoperativos y de inversión), costos variables y gastos administrativos variables. Los gastos preoperativos generales ascienden a 600 dólares y la inversión en activos fijos — tales como balanzas (de 2 y de 100 kilogramos), calculadora, pesamostos, cinta de $\mathrm{pH}$, baldes, mangueras, paleta para mover, embudo y tela de tocuyosuman 189 dólares, los que equivalen a 37.8 dólares mensuales si se considera una depreciación promedio anual de 20 por ciento.

Los costos variables suman 2094.45 dólares: se componen por la materia prima e insumos (1 994.45 dólares) y la mano de obra (100 dólares, se consideran cinco jornales de 20 dólares cada uno). Los costos de la materia prima e insumos incluyen uva, botellas, corchos, azúcar, capuchón, algodón, biopectinasa, bicarbonato de potasio, levadura, bisulfito de sodio y detergente. Un kilogramo de uva produce cerca de 650 mililitros de vino, dado que se requieren aproximadamente 800 kilogramos para producir 500 litros; entonces la uva representa cerca de $50 \%$ de los costos variables, como puede observarse en el cuadro 3.

Por su parte, los gastos administrativos variables incluyen los pagos de alquiler y servicios de luz y agua, seguros y registros, los cuales suman 58.48 dólares mensuales o 689.76 dólares anuales. El vino elaborado hasta esta etapa es totalmente apto para el consumo. Es el caso del vino económico. Sin embargo, para elevar su calidad es posible mejorar sus cualidades mediante el envejecimiento o crianza. 
Cuadro 3. Costo medio de producción para elaborar 500 litros de vino Premium empleando una tecnología artesanal, Baja California, 2012

\begin{tabular}{|lrc|}
\hline \multicolumn{1}{|c}{ Concepto } & \multicolumn{1}{c|}{ Dólares } & Por ciento \\
\hline Gastos preoperativos & 600.00 & 11.04 \\
Costos variables & 2094.45 & 38.52 \\
Gastos administrativos variables & 689.76 & 12.69 \\
Inversión en activos (20\%) & 37.80 & 0.70 \\
Barricas "tipo" & 2014.81 & 37.06 \\
Costos totales de producción & 5436.82 & 100.00 \\
\hline
\end{tabular}

Fuente: Elaboración del autor con base en Lagos et al. (2010) y Duarte, A. (2012).

Los vitivinicultores de Baja California enfrentan dificultades por la baja disponibilidad de agua. La escasez de agua en la región precisa a los productores a adoptar lo que Lee (2010) llama un enfoque holístico de sostenibilidad e impulsar cambios estructurales amplios. Para elevar la competitividad, los productores se orientan a nichos de mercado en los que ofertan un producto diferenciado, un vino de máxima calidad o Premium. Los productores buscan incorporar diversas mejoras y elementos distintivos en el proceso de producción con el objetivo de desarrollar sistemas de innovación en las empresas, como los sugeridos por Porter (1985) y Moguillansky et al. (2006). De igual manera, para mejorar el rendimiento y para reducir la incertidumbre del entorno promueven el coalinear la estrategia de la cadena de suministro y la estrategia competitiva por diferenciación de productos o por líderes de precios (Qi et al., 2011).

En la crianza, el tiempo de permanencia del vino en la barrica regularmente va de 6 a 18 meses, depende de la edad de las barricas. ${ }^{17}$ Cuanto más se alargue la crianza, en mayor medida se transmitirán al vino los caracteres de la madera, con la consiguiente pérdida de fruta. Para obtener un vino de calidad Premium es necesario al menos un envejecimiento de 12 meses en barricas nuevas. El costo de la barrica bordelesa de roble "tipo" (2/3 americana y 1/3 francés) es de 2014.81 dólares.

${ }^{17}$ Las barricas nuevas o con poco uso transmiten con mayor rapidez sus caracteres al vino que las viejas, ya que estas últimas han ido perdiendo sus aportes característicos con el uso y es necesaria una mayor permanencia del vino en ellas. 
Con 500 litros de vino se obtienen 666 botellas de 750 mililitros. De esta manera, al año 2012 el costo medio de producción de una botella de vino artesanal de calidad Premium es de 8.2 dólares y de 4.2 dólares el vino sin crianza. Al considerar una tasa de cambio mercantil de 13.2 pesos por dólar, el costo medio de producción de una botella de vino se ubica entre aquel sin crianza en barrica de 55 pesos y aquel de calidad Premium de 108 pesos.

\section{Distribuidoras de vinos de la región}

Del total de encuestas, 17 corresponden a distribuidoras de licores, seis son restaurantes y wine-bar y uno es una cadena comercial. Los resultados obtenidos indican que ofrecen vinos nacionales $(84.4 \%)$, básicamente de la región $(95.6 \%$ ) y solamente $4.4 \%$ de sus ventas proviene del resto del país. Los vinos importados representan $15.6 \%$ de sus ventas. Provienen principalmente de Chile (34.8\%), Argentina (21.7\%), España (17.4\%) y de Estados Unidos $(8.7 \%)$. Por tipos de vino, el tinto posee la mayor participación en las ventas $(75.5 \%)$, las varietales preferentes son Cabernet Sauvignon (51.1\%), Merlot (18.0\%), Tempranillo (16.4\%), Petite Syrah (3.6\%) y mezcla de tintos (3.6\%). En menor cuantía contribuye el vino blanco (15.9\%). Las varietales principales son Chardonay (26.9\%), Chenin Blanc (25.0\%), mezcla de blancos (16.4\%), Sauvinong Blanc (13.6\%) y Colombard (9.5\%). Los vinos rosados tienen un reducida participación en el mercado (8.6\%), las varietales más comúnmente referidas son mezcla $(29.4 \%)$, Grenach (28.2\%), Zinfandel Blanc (16.3\%), Zinfandel (13.9\%) y Merlot (2.9\%).

Referente a los precios de los vinos, en $79.2 \%$ de las ventas la botella cuesta más de 150 pesos; sólo en $12.5 \%$ de las ventas la botella cuesta menos de 100 pesos. Los vinos tintos son más caros que los blancos, pero el rango de precios es muy amplio; son hasta $50 \%$ más caros que los blancos, según indica cerca de $80 \%$ de los encuestados. ${ }^{18}$

${ }^{18}$ Font, Gudiño y Sánchez (2009, pp. 12-13) indican que los vinos mexicanos, en precios, se ubican en desventaja en relación con los extranjeros; los más económicos y de calidad están entre 70 y 80 pesos mexicanos, mientras que se pueden encontrar vinos españoles y chilenos desde 55 pesos. Los vinos blancos y tintos mexicanos van de los 70 a los 100 pesos, mientras que los blancos y tintos chilenos, argentinos o españoles, 
En cuanto a las estrategias de mercadotecnia, cerca del $74 \%$ de las distribuidoras de vinos tienen publicidad en un sitio web. No obstante ello, alrededor de $20 \%$ de los encuestados indica que para posicionar el vino de la región es necesario promover una mayor publicidad, explorar nuevos mercados o impulsar la cultura del consumo del vino a través de talleres.

En relación con la comercialización del vino producido en la región, destaca el hecho de que la tercera parte de las distribuidoras de vino enfrentan problemas importantes de abastecimiento del producto en los rubros de tiempo de entrega, cantidad de producto y capacitación de personal encargado de la distribución. También indicaron que, aunque poco, existe intermediarismo entre los productores y los distribuidores de vino. Ello hace evidente la necesidad de mejorar la cadena de suministro en al menos dos sentidos, los fabricantes y minoristas deben incluir una "respuesta precisa" tanto en la predicción de la planificación como en todo el proceso de producción (Fischer et al., 1994). También, la cadena de suministro debe atender los conceptos de la triple "A": agilidad, adaptabilidad y alineación (Lee, 2004).

A decir de cerca de la mitad de los distribuidores de vino, los altos impuestos junto con los precios altos del vino producido en la región son una limitante en las ventas del producto. Por lo que apoyos gubernamentales a la industria vitivinícola, tales como la disminución de impuestos, otorgar facilidades para cumplir con obligaciones fiscales ante hacienda, financiamiento a la producción, entre otros, son acciones necesarias que ayudan a posicionar en el mercado los vinos producidos en la región.

\section{Problemática enfrentada por los productores de vino de Baja California}

En el desarrollo de la vitivinicultura los productores de Baja California enfrentan una amplia diversidad de problemas que bien pueden separarse

van desde los 55 hasta los 100 pesos. En vinos de calidad superior (tintos de reserva), los mexicanos tienen precios que van de los 140 a los 500 pesos, los extranjeros van desde 115 hasta 300 pesos. En vinos considerados de máxima calidad la diferencia de precios no es muy grande: los vinos mexicanos están entre 500 y 1000 pesos, mientras que los extranjeros van de los 325 a poco más de 1200 pesos. 
en dos grupos: los internos de la empresa y los institucionales o externos a ella. En los primeros destacan diferentes debilidades como falta de recursos económicos, necesidad de modernos equipos y maquinaria, poder de negociación y capacidad en la distribución del producto, entre otros.

Por su parte, los problemas institucionales o externos a la empresa se refieren a los que afectan a un nivel global al sector vitivinícola; en éstos los productores enfrentan la escasez de agua en la región, lo que repercute en la salinización de los suelos y en la calidad de los vinos; la falta de proveedores locales lo que redunda en altos precios de los insumos; el escaso financiamiento gubernamental a la actividad, y las elevadas tasas impositivas de un nivel similar a las que se les cargan a otras bebidas de mayores concentraciones alcohólicas, como las destiladas. La atención de estas restricciones precisa de una participación amplia y de una respuesta organizada de los empresarios, el sector académico y de investigación, así como de los tres niveles de gobierno. El cuadro 4 muestra un resumen de los principales problemas de los vitivinicultores.

\section{Conclusiones}

En la región vitivinícola de Baja California se produce alrededor de $90 \%$ del vino que se consume a nivel nacional. Son poco más de medio centenar las casas comerciales productoras de vino. El análisis de la cadena de valor del vino es útil para elevar la competitividad regional a través de la reducción de costos y la diferenciación del producto. En este trabajo se construye el esquema tipo de diagrama de flujo horizontal de la cadena de valor del vino, el cual resultó útil para identificar aciertos y deficiencias en las etapas del proceso productivo y para proponer las acciones correctivas que deberá impulsar el responsable en cada una de las etapas.

Con base en el volumen y en las características de la técnica de producción, las empresas productoras de vino bajacaliforniano se diferencian en cuatro grandes grupos: las empresas grandes son las que, en todo el proceso de elaboración del vino, usan técnicas modernas y producción de escala, algunas importan del extranjero una parte significativa del mosto o zumo; las medianas combinan técnicas artesanales y modernas en la producción; mientras que las pequeñas y las microempresas usan técnicas artesanales 


\section{Cuadro 4. Cadena de valor del vino en Baja California: Problemática enfrentada por los productores, 2012}

\begin{tabular}{l} 
Producción de uva \\
- Falta de agua* \\
- Hay depredadores (los pájaros consumen el fruto) \\
- En la comercialización del producto existe intermediarismo \\
\hline Producción de vino \\
- Inversión inicial fuerte (el capital propio es insuficiente, se requiere financiamiento) \\
- Cultura y conocimientos (falta de experiencia, son necesarios cursos de capacitación) \\
- Maquinaria y equipo (son insuficientes y también falta modernizarlos) \\
- Falta de espacios (en planta, en cavas, entre otros) \\
- Comercialización (financiamiento, conocimiento de mercados y canales) \\
para acceder a ellos) \\
Proveeduría de insumos* \\
- Falta de proveedores locales \\
- Precios altos de insumos \\
\hline Institucionales* \\
- Banca de desarrollo (financiamiento insuficiente) \\
- Tasas impositivas elevadas
\end{tabular}

Notas: * Problemas externos a la empresa.

Fuente: Elaboración del autor con base en González (2012, p. 34).

de producción de vino, pero también, en algunos casos, maquilan segmentos del proceso de producción a las empresas medianas y grandes.

El costo medio de producción de vino bajacaliforniano empleando una tecnología artesanal fluctúa entre 55 pesos la botella de vino sin crianza en barrica y hasta 108 pesos la botella de vino de calidad Premium.

Los distribuidores de vino bajacaliforniano enfrentan los problemas principales de abastecimiento del producto en los rubros de tiempo de entrega, cantidad de producto y capacitación de personal encargado de la distribución. Adicionalmente, ayudaría a posicionar los vinos producidos en la región la aplicación de acciones gubernamentales como la disminución de impuestos, facilidades para cumplir con las obligaciones fiscales ante hacienda, así como esquemas competitivos de financiamiento.

Con base en la estimación empírica de la cadena de valor de las vitivinícolas de Baja California, se pueden concebir al menos dos conclusiones: 
la primera es que la herramienta de cadena de valor está directamente relacionada con el valor agregado de un producto, aquello que el cliente está dispuesto a pagar por los productos de la empresa y que permite medir su posición competitiva. En este sentido, es fundamental para las casas vitivinícolas el contar con esta herramienta de análisis, ya que permite realizar un estudio completo de los procesos de producción de insumos y de productos, así como de la gestión y el desarrollo de bienes derivados de la uva.

La segunda es que con base en el análisis de la cadena de valor se identificaron los principales problemas a los que deben orientarse acciones correctivas, tanto internos en las empresas como aquellos que enfrentan a nivel del propio sector. Destacan las restricciones que se refieren a infraestructuras productivas, financiamiento, proveedores locales, comercialización, formación y capacitación de recursos humanos, falta de agua, impuestos altos, entre otros. La superación de tales restricciones, acompañada con la introducción de mejoras en los procesos productivos y la identificación de nuevos nichos de mercado, entre otras acciones, son elementos clave para lograr mayor participación del vino bajacaliforniano en los mercados nacional e internacional.

\section{Bibliografía}

Consejo Mexicano Vitivinícola (cmv). (2010). Consejo Mexicano Vitivinícola. Recuperado del sitio de Internet del Consejo Mexicano Vitivinícola: http:// vinoclub.com.mx/index.php? module $=$ Articulos\&aid $=67$

Consejo Mexicano Vitivinícola (cmv). (2012). Economía y mercados: Mercado de vino en México. Recuperado del sitio de Internet del Consejo Mexicano Vitivinícola: http://www.uvayvino.org/index.php/eonomia

Cooke, G. y Lapsley, J. (1988). Home Winemaking: Making Table Wine at Home. Oakland, California: Viticulture \& Enology, University of California.

Distrito de Desarrollo Rural (DDR). (2011). Entrevista a DDR 001-Ensenada, área de Extensión Agrícola/Entrevistador: S. González. Costos de producción por hectárea de uva en la etapa de mantenimiento y en la modalidad de riego, ciclo 2009-2009. Secretaría de Agricultura, Ganadería, Desarrollo Rural, Pesca y Alimentación (Sagarpa). Copia en posesión del autor.

Duarte, A. (2012). Precios de artículos para vinícolas y oliveras. Entrevista a A. Duarte/ Entrevistador: S. González. Distribuidora de artículos para vinícolas y oliveras (DAVO). Copia en posesión del autor. 
Fischer, M., Hammond, J., Obermeyer, W. y Raman, A. (Mayo-junio, 1994). Making Supply Meet Demand in an Uncertain World. Harvard Business Review, 72(3), 83-93.

Font, I., Gudiño, P. y Sánchez, A. (2009). La industria vinícola mexicana y las políticas agroindustriales: panorama general. Redpol Estado, Gobierno y Políticas Públicas, (2), 1-30.

Food and Agriculture Organization of the United Nations (FAO). (2013). FAOSTAT: Trade. Recuperado de http://faostat.fao.org/

Galitsky, C., Worrell, E., Radspieler, A., Healy, P. y Zechiel, S. (2005). Best Winery Guidebook: Benchmarking and Energy and Water Savings Tool for the Wine Industry. Berkeley, California: Energy Analysis Department, Environmental Energy Technologies Division, Ernest Orlando Lawrence Berkeley National Laboratory, University of California.

Girán, R., Vargas, H. y Osta, K. (2008). Propuesta metodológica para el análisis de competitividad empresarial en la pequeña y mediana agroindustria alimentaria. Revista Ingeniería Industrial, 7(1), pp. 5-14.

González, S. (2012). Cadena de valor del vino en los valles vitivinícolas de Baja California. En Autor (Coord.), Plan de acción para la innovación y competitividad de los valles vitivinícolas de Baja California. Tijuana, Baja California: Proyecto Conacyt-Fordecyt, El Colegio de la Frontera Norte.

Iglesias, D. (Febrero, 2002). Cadenas de valor como estrategia: Las cadenas de valor en el sector agroalimentario. La Pampa, Argentina: Estación Experimental Agropecuaria Anguil, Instituto Nacional de Tecnología Agropecuaria.

Lagos, J., Espinoza, C. y Rojas, W. (2010). Proceso y estructura de costo de producción. Recuperado de http://www.slideshare.net/jaldon/proceso-yestructura-de-costo-de-produccin

Lee, H. L. (Octubre, 2004). The Triple-A Supply Chain: The Triple-A Supply Chain. Harvard Business Review, 102-122.

Lee, H. L. (Octubre, 2010). Don't Tweak You Supply Chain-Rethink It End to End. Harvard Business Review, 63-69.

Lundy, M., Gottret, M. V., Cifuentes, W., Ostertag, C. F. y Best, R. (2004). Diseño de estrategias para aumentar la competitividad de cadenas productivas con productores de pequeña escala: Manual de campo. Colombia: Proyecto de desarrollo de agroempresas rurales CIAT.

Mendoza, A. A. (2011). Vinificaciones clásicas y no convencionales. Revista Enología, (1), 1-16.

Moguillansky, G., Salas, J. C. y Cares, G. (2006). Capacidad de innovación en industrias exportadoras de Chile: la industria del vino y la agroindustria hortofrutícola (núm. 79). Santiago de Chile: cepal, Serie comercio internacional. 
Ponce, A. (2009). La fermentación del vino. La química entre dos. Recuperado de http://www.articuloz.com/vino-articulos/la-fermentacion-del-vino-la-quimicaentre-dos-964223.html

Porter, M. (1985). Competitive Advantage: Creating and Sustaining Superior and Performance. Nueva York: The Free Press.

Qi, Y., Zhao, X. y Sheu, C. (2011). The Impact of Competitive Strategy and Supply Chain Strategy on Business Performance: The Role of Environmental Uncertainty. Decision Sciences Journal, 42(2), 371-389.

Sánchez, S. (2002). La competitividad en el sistema productivo local del vino en el Valle de Guadalupe (Tesis de maestría). El Colegio de la Frontera Norte, Tijuana, México.

Secretaría de Fomento Agropecuario (Sefoa), Oficina Estatal de Información para el Desarrollo Rural Sustentable. (2011). Estudio estadístico sobre producción de uva en Baja California. Baja California: Gobierno del Estado de Baja California, Sagarpa, OEIDRus.

Sepúlveda, J. (2009). Aspectos geográficos y estadísticos de la viticultura del estado de Baja California. Ensenada, Baja California: Sistema Producto Vid de Baja California y Fundación Produce de Baja California.

Sistema Producto Vid de Baja California (SPV). (s/f). Información general del clúster. Recuperado del sitio de Internet de Sistema Producto Vid de Baja California: http://vidyvino.org/docs/SPVid_Info_Cluster.pdf

Sistema Producto Vid de Baja California (sPv). (2008). Información general del clúster. Recuperado del sitio de Internet de Sistema Producto Vid de Baja California: http://vidyvino.org/docs/SPVid_Info_Cluster.pdf

Vázquez, A. (2002). Endogenous development: Networking, innovation, institutions and cities. Londres: Routledge.

Vázquez, A. (2005). Las nuevas fuerzas del desarrollo. España: Antoni Bosch Editor. Vivanco, M., Martínez, F. y Taddei, I. (2010). Análisis de competitividad de cuatro sistema-productos estatales de Tilapia en México. Revista Estudios Sociales, 18(35), 166-207. 